\title{
Morfología del Arco Maxilar y Mandibular en Niños de Ascendencia Mapuche y no Mapuche
}

\author{
Morphology of the Maxillary and Mandibular Arch in Children \\ of Mapuche and Non-Mapuche Ancestry
}

Paulina Agurto S. \& Paulo Sandoval V.

AGURTO, S. P. \& SANDOVAL, V. P. Morfología del arco maxilar y mandibular en niños de ascendencia Mapuche y no Mapuche. Int. J. Morphol., 29(4):1104-1108, 2011.

RESUMEN: La forma de arco ideal se ha tratado de determinar por muchos autores, sin llegar a una forma única, debido a que existen variaciones en un mismo individuo, por género, raza y factores ambientales. Se realizó un estudio de corte transversal, en una muestra no probabilística de 40 niños de ascendencia mapuche y 35 niños no mapuche, en los cuales se determinó la forma de arco maxilar y mandibular a través de una regla morfométrica con las formas de arco triangular, ovoídea y cuadrada (Orthoform I, II y III). En el análisis de las formas de arco para cada grupo y su asociación, se observaron algunas diferencias en la distribución de las formas cuadrada y triangular, pero no son estadísticamente significativas. Respecto de la coincidencia de las arcadas se encontró para los mapuches un Índice de Kappa de 0,3096 y para los no mapuches de 0,1204, lo que indica que en ambos grupos no existe concordancia. Existe diferencia en la forma de arco entre mapuches y no mapuches. La forma predominante en ambos grupos es la ovoídea. Existe baja coincidencia de la forma de arco maxilar y mandibular en un mismo individuo.

PALABRAS CLAVE: Arco dental; Grupo étnico.

\section{INTRODUCCIÓN}

La forma de arco ideal se ha tratado de determinar desde los inicios de la ortodoncia. Existen variaciones interindividuales asociadas a género, raza, influencias ambientales, entre otros, y a lo largo del crecimiento en un mismo individuo. En 1885, Bonwill notó la forma trípode de la mandíbula, que forma un triángulo equilátero con la base extendida de cóndilo a cóndilo y los lados de cada cóndilo a la línea media de los incisivos centrales (Bonwill, 19841985). Hawley (1905), usó algunos de los principios de Bonwill, proponiendo un método geométrico para la construcción de la forma ideal de arco. Black (1902) estableció que los dientes superiores están ordenados en una semi-elipse y que los dientes inferiores están ordenados similarmente en una pequeña curva. Broomell (1902), en el mismo año dijo que "los dientes están ordenados en los maxilares en la forma de dos curvas parabólicas".

En 1907, Angle discutió en detalle la "línea de oclusión", que él definió como "la línea con la cual, de acuerdo al tipo en forma y posición, los dientes deben estar en armonía aunque en oclusión normal”. Dijo, que la forma de ésta línea se parecía a una curva parabólica, pero una que varía muchísimo debido a raza, tipo, temperamento, etc. (Angle, 1907).

El texto Gray's Anatomy en 1942, estableció: “El arco dental maxilar forma una curva elíptica y el arco dental mandibular forma una curva parabólica" (Johnston \& Whillis, 1942).

En 1934, Chuck destacó la variación en la forma de arco humano y fue el primero en clasificar las formas de arco como cuadrada, redonda, oval y estrecha (Chuck, 1934). Así, a través de los años las formas de arco de Bonwill-Hawley han sido las formas de arco más consistentemente usadas como plantilla de inicio para los ortodoncistas de arco de canto. En 1949, MacConaill \& Scher establecieron que considerando la línea de oclusión, sería imposible para una elipse y una parábola encontrarse en uno o cualquier punto. El concluyó, que la descripción elipse-parábola de los dos arcos dentales, aunque elegante, no tiene relación inmediata a la función. Estableció, que una buena y bien sabida curva, la curva catenaria, calza en muchos casos con exactitud por lo que podía ser con- 
siderada como la "curva ideal" de oclusiones comunes. La curva catenaria fue formada simplemente por la suspensión de una cadena de largo adecuado desde dos puntos de ancho variable (por ejemplo, el ancho de los molares más distales en la forma de arco) (MacConaill \& Scher, 1949). Scott (1957), Burdie \& Lillie (1966) y Musich \& Ackerman (1973) apoyaron la forma de curva catenaria como forma de arco ideal.

Brader (1972) respecto a la geometría de la curva de la forma de arco dental dijo ser la mejor aproximación a una curva cerrada con las propiedades inherentes en la elipse trifocal, con los dientes ocupando sólo la porción del extremo estrecho de la curva. Recomendó un arco guía con cinco formas de arco. La selección de la forma de arco adecuada se basó en el ancho de arco a nivel del segundo molar medido en la cara lingual a nivel de la superficie gingival.

Izard (1927) se basó en el método de predeterminación de arco sobre las proporciones entre el ancho del arco y la profundidad facial; concluyó que aproximadamente un $75 \%$ de las formas de arco fueron representadas por una elipse, $25 \%$ por una parábola y $5 \%$ por una forma de "U".

El año 2000, Burris \& Harris publicaron un artículo de la "Forma y tamaño del arco maxilar en Negros y Blancos americanos"; encontraron mayor cantidad de arcos cuadrados en los negros y triangulares en los blancos, sin diferencias por sexo (Burris \& Harris, 2000).

En el 2004, Guerrero \& Améstica, realizaron un estudio de la forma de arco mandibular en una muestra de 103 jóvenes chilenos, usando las formas estrecha, ovoídea y cuadrada (3M, Unitek) y los resultados obtenidos fueron: $26 \%$ estrechas, $35 \%$ cuadradas y $39 \%$ ovoides (Guerrero \& Améstica, 2004).

Kook et al. (2004) realizaron una comparación de las formas de arco entre población coreana y norteamericana blanca, encontrando que en los coreanos predominaba la forma cuadrada $46,7 \%$, seguida de la ovoídea con un $34,5 \%$ y la estrecha con un $18,8 \%$. Mientras, que en el grupo blanco la forma predominante fue la estrecha con un $43,8 \%$, seguida de la ovoide con $38,1 \%$ y luego la cuadrada con $18,1 \%$.

Rivera et al. (2008) estudiaron la forma y tamaño de los arcos dentales en una población escolar de indígenas amazónicos y encontraron un $86 \%$ de forma ovoídea y $14 \%$ cuadrada en el maxilar y un $75 \%$ ovoídea y $25 \%$ cuadrada en la mandíbula.

El propósito de este estudio, es describir la forma de arco presente en una población de la novena región en Chile, en niños con ascendencia mapuche y no mapuche.

\section{MATERIAL Y MÉTODO}

Se realizó un estudio descriptivo, de corte transversal, en un total de 75 niños Mapuches y no Mapuches, de ambos sexos. La muestra de la etnia Mapuche corresponde a un total de 40 niños, de entre 10 y 12 años, pertenecientes al Servicio Dental del Hospital Intercultural Maquehue. Se determinó la etnia mapuche desde el punto de vista antropológico por métodos cualitativos, a aquellos individuos con más de un apellido en lengua mapuche y autorreconocimiento de pertenencia a la etnia. No se consideró la caracterización genética a través de grupos sanguíneos, ya que, existe evidencia para señalar que no es lo suficientemente sensible y específica (Cifuentes et al., 1988).

La muestra no mapuche fue seleccionada de pacientes atendidos en la Clínica de Odontopediatría Docente Asistencial de la Escuela de Odontología de la Universidad de la Frontera, y corresponde a una muestra de 35 pacientes, cuyas edades fluctúan entre los 9 y 12 años.

Todos estos pacientes debían tener los dientes erupcionados por completo hasta el primer molar permanente, ausencia de malformaciones que comprometiesen el arco dentario y sin historia de tratamiento de ortodoncia previo.

La unidad de análisis corresponde a 150 modelos de estudio, confeccionados en yeso piedra, de ambas arcadas de las muestras anteriormente señaladas.

Cada modelo fue clasificado de acuerdo a su forma por un examinador previamente calibrado. Se consideraron tres formas de arco; la triangular, cuadrada y ovoídea, por lo que se utilizó como referencia las plantillas de la 3M Unitek (Orthoform Templates Diagnostics Set), que considera como Orthoform I a la arcada de forma triangular, Orthoform II a la cuadrada y Orthoform III a la ovoídea (Fig. 1).

El análisis estadístico utilizado para determinar la asociación en la distribución de frecuencias de las formas de arco maxilar y mandibular para la muestra mapuche y no mapuche, fue el Test Chi-cuadrado.

La determinación de la coincidencia interarcadas de cada individuo de ambas muestras, se realizó a través del Test de McNemar, que mide el grado de concordancia por medio del Índice Kappa. El nivel de significancia establecido fue $(\mathrm{P}<0,05)$.

Todos los cálculos estadísticos fueron realizados a través del paquete estadístico SAS (versión 9.1, SAS, Cary, NC). 


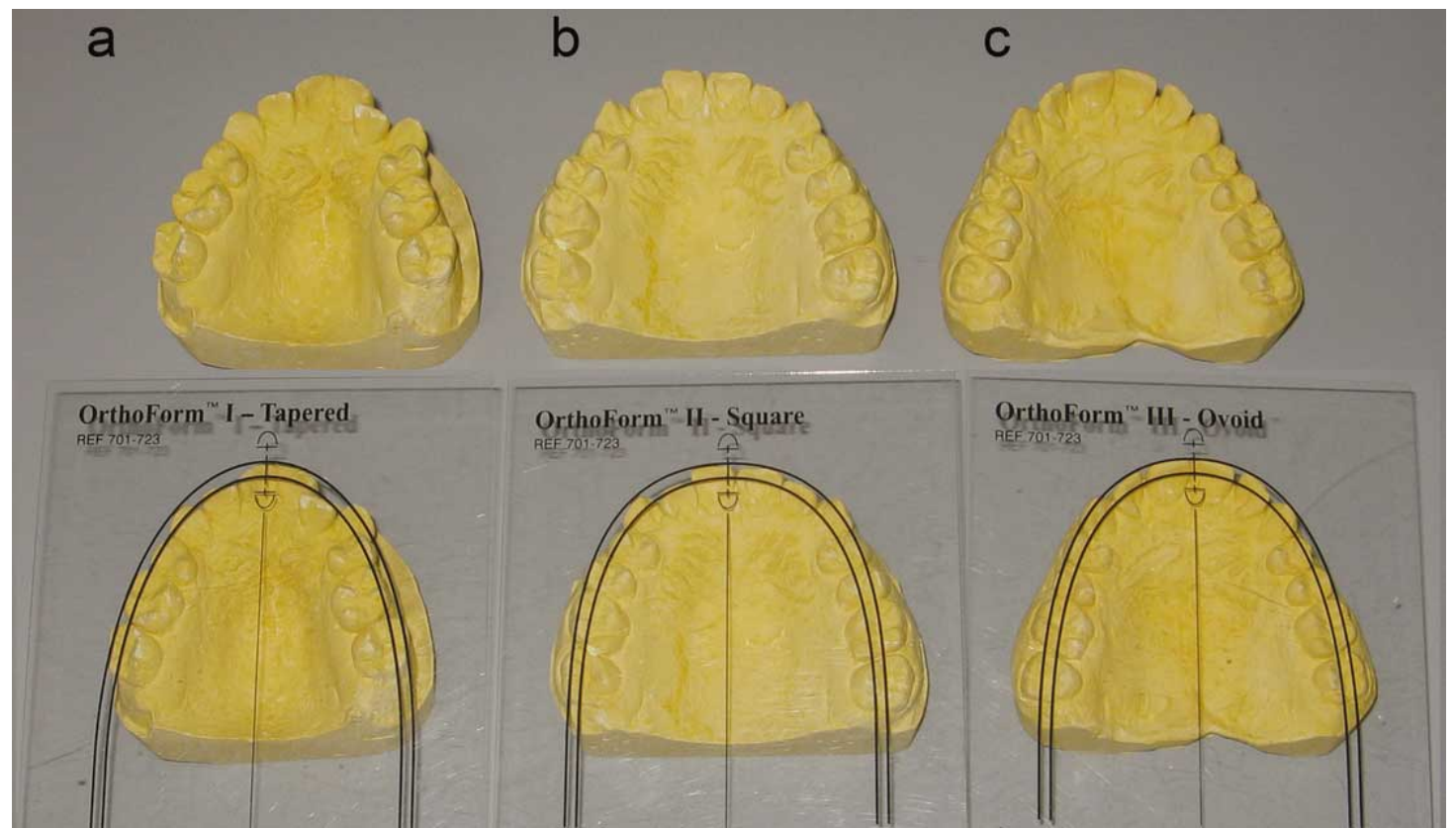

Fig. 1. Clasificación de la forma de arco con plantillas morfométricas. a) Triangular (Orthoform I), b) Cuadrada (Orthoform II) y c) Ovoídea (Orthoform III).

\section{RESULTADOS}

Los resultados observados por arcada fueron los siguientes: En la maxila en la muestra mapuche se encontró un 17,5\% Orthoform I, un 27,5\% Orthoform II y más de la mitad correspondían a Orthoform III con un 55\%. En la muestra no

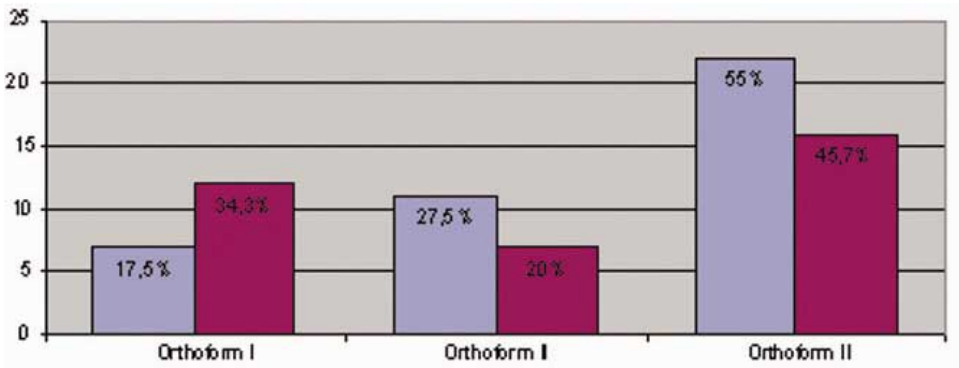

Fig. 2. Asociación de las formas de arco maxilar en niños mapuches (10 -12 años) y no mapuches (9 -12 años).

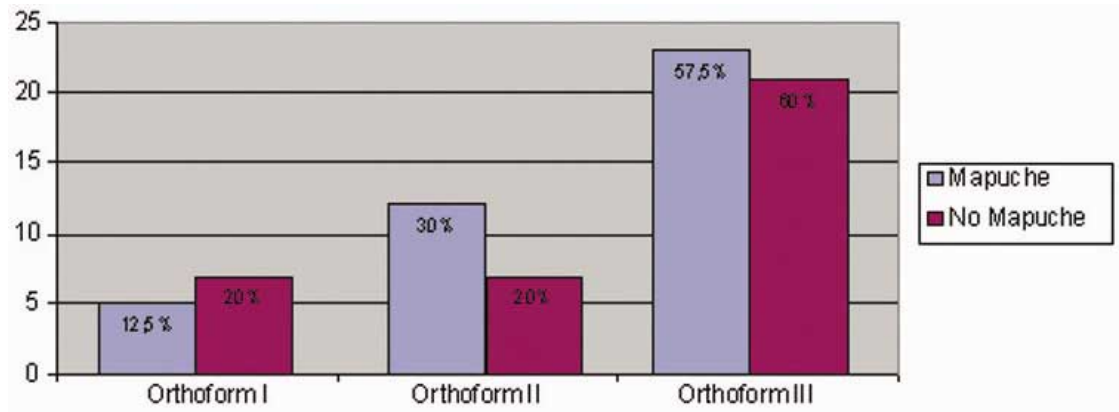

Fig. 3. Asociación de las formas de arco mandibular en niños Mapuches (10 - 12 años) y no mapuches (9 -12 años). mapuche se encontró un $34,3 \%$ Orthoform I, un 20\% Orthoform II y un $45,7 \%$ de Orthoform III, observándose en este grupo casi el doble de arcadas Orthoform I en comparación al grupo mapuche $(\mathrm{P}=0,2428)$ (Figura 2).

En la mandíbula en el grupo de niños mapuches, se encontró: $12,5 \%$ para Orthoform I, 30\% de Orthoform II y $57,5 \%$ de Orthoform III. En el grupo no mapuche se halló igual proporción para Orthoform I y II con un $20 \%$ y un $60 \%$ para Orthoform III $(\mathrm{P}=0,4934)$ (Figura 3).

Se analizó también la coincidencia total de la forma de arco para ambos grupos y se encontró mayor coincidencia en la muestra mapuche con un $60 \%$ en comparación a la no mapuche con un porcentaje de coincidencia $45,7 \%$. Cabe destacar los altos porcentajes de no coincidencia para ambos grupos, cuyos valores se especifican en la Tabla I. 
Tabla I. Coincidencia total inter-arcadas para ambos grupos.

\begin{tabular}{lcccccc}
\hline \multicolumn{7}{c}{ Coincidencia } \\
\hline Etnia & $\mathrm{Si}$ & $\%$ & No & $\%$ & Total & $\%$ \\
Mapuche & 24 & 60,0 & 16 & 40,0 & 40 & 100 \\
No Mapuche & 16 & 45,7 & 19 & 54,3 & 35 & 100 \\
\hline Total & 40 & 53,3 & 35 & 46,7 & 75 & 100 \\
\hline
\end{tabular}

$53 \%$ de la muestra, comparado a la población blanca o caucásica donde estas dos formas constituían más del $80 \%$ y la forma cuadrada sólo un $18,1 \%$. Guerrero \& Améstica, en un estudio en población chilena, encontró distribuciones similares para las tres formas de arco estudiadas en la mandíbula (ovoide 39\%, cuadrada $35 \%$ y $26 \%$ triangular o estre-

Al aplicar el Test de McNemar para cada grupo por separado, para la muestra mapuche se encontró un Índice de Kappa de 0,3096 y para los no mapuche 0,1204, lo que indica que no hay acuerdo entre las medidas, es decir, tanto en la muestra mapuche como en la no mapuche, hubo un bajo porcentaje de coincidencia de ambas arcadas de cada individuo en estudio.

\section{DISCUSIÓN}

La población mapuche es la etnia originaria de la IX región y se encuentra ampliamente distribuida a lo largo de Chile. Existen pocos estudios respecto a sus características craneofaciales y las diferencias con el resto de la población, es por esto, que se busca obtener más información respecto a la forma del arco dental de estos individuos y compararlos con la población no mapuche; antecedentes que pueden ser importantes de considerar en la planificación del tratamiento ortodóncico.

En este estudio se encontraron diferencias en la distribución de la forma del arco al comparar ambos grupos. En la maxila, se encontró una mayor proporción de arcos triangulares (Orthoform I) en el grupo no mapuche con un 34,3\%, que casi duplica a lo encontrado en el grupo mapuche. Se encontró además que en este último grupo había un $10 \%$ más de arcos cuadrados (Orthoform II). Sin embargo, para la forma ovoídea los porcentajes son similares. Estos hallazgos son semejantes a las diferencias observadas en el arco maxilar por Burris \& Harris, que encontró mayor cantidad de arcos cuadrados y menos arcos triangulares al comparar la población negra americana con la población blanca.

En la mandíbula se encontró que para ambos grupos predominaba la forma ovoídea $(58,7 \%$ promedio), no obstante, la población mapuche posee más arcos cuadrados (atribuibles a su origen asiático), al compararlos con los que no tienen ascendencia mapuche (30\% vs 20\%). Kook et al. en un estudio comparativo de población asiática (coreana) con sujetos de raza blanca, estableció que en la población coreana predominaban los arcos de forma cuadrada con $46,7 \%$ y las formas ovoídea y triangular consistían en un cha), diferente a lo encontrado en nuestro estudio en el grupo no mapuche donde predominó la forma ovoídea (60\%).

En un estudio realizado en una población escolar de indígenas amazónicos en Colombia Rivera et al., la forma predominante fue la ovoídea, ya que, en los arcos superiores el $86 \%$ de los niños tenían forma ovalada, en tanto que, el $14 \%$ presentaban forma cuadrada; para el arco inferior las medidas promedio fueron $75 \%$ ovalados y $25 \%$ cuadrados, no reportaron datos para la forma triangular.

Al comparar la coincidencia de ambas arcadas en un mismo individuo, no se encontraron otros estudios similares. Los valores encontrados en este estudio muestran que para ambos grupos la coincidencia es sólo un poco superior al 50\%, lo que nos indica, que no sólo se deben analizar las diferencias interpersonales de las formas de arco en los pacientes por su origen, etnia o biotipo, si no que hay que evaluar además, que en un mismo individuo puede haber diferencias.

Los autores consideramos este estudio como un piloto, estimando necesario realizar una nueva investigación que cuente con un muestreo probabilístico adecuado, que incluya segmentos representativos por edad en dentición temporal, mixta y permanente.

En este estudio se pudo comprobar que existen variaciones en la distribución de las formas de arco, tanto en un mismo individuo como en la población general, diferencias principalmente dadas por la etnia u origen racial.

En la población mapuche se observó mayor proporción de arcos cuadrados, tanto en el maxilar como en la mandíbula, al ser comparado con el grupo no mapuche, sin embargo, para ambos grupos la forma predominante es la ovoídea.

Estos hallazgos pueden ser de gran importancia clínica, en la realización de un diagnóstico y tratamiento adecuado en nuestros pacientes, que nos permitan determinar e individualizar los arcos dentarios (McLaughlin \& Bennett, 1999; McLaughlin et al., 2001), para no modificar su forma evitando el riesgo de recidiva posterior al tratamiento de ortodoncia. 
AGURTO, S. P. \& SANDOVAL, V. P. Morphology of the maxillary and mandibular arch in children of Mapuche and Non-Mapuche ancestry. Int. J. Morphol., 29(4):1104-1108, 2011.

SUMMARY: The form of determination of ideal arch has been attempted by many authors, without reaching only one form, as variations exist in the same individual for sex, race and environmental factors. A cross sectional study was carried out in a nonprobabilistic sample of 40 Mapuche children and 35 Non-Mapuche children in which the maxillary and mandibular form was determined through morphometric ruler with forms of triangular, ovoid and square arch (Orthoform I, II and III). In the analysis of arch forms for every group and its association, some differences were observed in the square and triangular distribution of the forms, these are however not statistically significant. In reference to the coincidence of the arcades was for the Mapuche Kappa Index of 0.3096 and for NonMapuche of 0.1204 indicating that concordance does not exist in both groups. There are differences in the form of arch between Mapuche and Non-Mapuche. The predominant form in both groups is the ovoid form. There is a lesser coincidence of maxillary and mandibular form to jaw arch in the same individual.

KEY WORDS: Dental arch; Ethnic groups

\section{REFERENCIAS BIBLIOGRÁFICAS}

Angle, E. H. Treatment of malocclusion of the teeth. $7^{\text {th }}$ ed. Philadelphia, Philadelphia, SS White Dental Manufacturing Co., 1907

Bonwill, W. G. A. Geometrical and mechanical laws of articulation. Tr. Odont. Soc. Penn., 119-33, 1884-1885.

Black, G. V. Descriptive anatomy of the human teeth. $5^{\text {th }}$ ed. Philadelphia, Philadelphia, SS White Dental Manufacturing Co., 1902. pp.130-52.

Brader, A. C. Dental arch form related with intraoral forces: PR=C. Am. J. Orthod., 61(6):541-61, 1972.

Broomell, I. N. Anatomy and histology of the mouth and teeth. $2^{\text {nd }}$ ed. Philadelphia, P. Blakiston's Son \& Co., 1902. p.99.

Burdi, A. R. \& Lillie, J. H. A catenary analysis of the maxillary dental arch during human embryogenesis. Anat. Rec., 154(1):13-20, 1966

Burris, B. G. \& Harris, E. F. Maxillary arch size and shape in American blacks and whites. Angle Orthod., 70(4):297-302, 2000 .

Cifuentes, L.; Valenzuela, C. Y.; Cruz-Coke, R.; Armanet, L.; Lyng, C. \& Harb, Z. Genetic characterization of the hospital population of Santiago, Chile. Rev. Med. Chile, 116(1):28-33, 1988.

Chuck, G. C. Ideal arch form. Angle Orthod., 4:312-27, 1934.

Guerrero, K. \& Améstica R. Estudio clínico de formas de arco mandibular en jóvenes chilenos. Tesis pata optar al Título de Cirujano Dentista, Escuela de Odontología, Universidad de Talca, 2004.

Hawley, C. A. Determination of the normal arch and its application to orthodontia. Dent. Cosmos, 47:541-52, 1905.
Izard, G. New method for the determination of the normal arch by the function of the face, Int. J. Orthodontia, 13:582-95, 1927.

Johnston, T. B. \& Whillis, J. (Eds). Gray's Anatomy. $28^{\text {th }}$ ed. London, Longmans Green \& Co., 1942.

Kook, Y. A.; Nojima, K.; Moon, H. B.; McLaughlin, R. P. \& Sinclair, P. M. Comparison of arch forms between Korean and North American white populations. Am. J. Orthod. Dentofacial Orthop., 126(6):680-6, 2004.

MacConaill, M. A. \& Scher, E. A. The ideal form of the human dental arcade, with some prosthetic application. Dent. Rec., 69(11):285-302, 1949.

McLaughlin, R. \& Bennett, J. Arch form considerations for stability and esthetics. Rev. Esp. Orthod., 29(2):46-63, 1999.

McLaughlin, R.; Bennett, J. \& Trevisi, H. Mecánica sistematizada del tratamiento ortodóncico. Madrid, Mosby, 2001. pp.77-8.

Musich, D. R. \& Ackerman, J. L. The catenometer: A reliable device for estimating dental arch perimeter. Am. J. Orthod., 63(4):366$75,1973$.

Rivera, S.; Triana, F.; Soto, L. \& Bedoya, A. Forma y tamaño de los arcos dentales en una población escolar de indígenas amazónicos. Colomb. Med., 39(1):51-6, 2008.

Scott, J. H. The shape of the dental arches. J. Dent. Res., 36:9961003, 1957.

Dirección para correspondencia:

Mg. Paulo Sandoval Vidal

Departamento de Odontología Integral

Facultad de Medicina, Universidad de La Frontera

CHILE

Email: hpsandov@ufro.cl

Recibido : 26-07-2011

Aceptado: 28-08-2011 